\title{
MODELAÇÃO PROATIVA DE METAS \\ E ELABORAÇÃO DE SOLUÇÕES INFORMATIZADAS
}

Sandra Vieira ${ }^{1}$

\begin{abstract}
RESUMO
Soluções informatizadas para a resolução de problemas que emergem de necessidades cotidianas concretas são estruturadas através de algoritmos dedutivos. Todavia, antes mesmo de elaborar algoritmos, há uma etapa abdutiva guiada pela meta a ser alcançada que é geralmente negligenciada. Seguindo a Teoria da Conciliação de Metas proposta por Rauen (2013), pretende-se descrever e explicar neste artigo como ocorre o processo de elaboração, execução e checagem de hipóteses abdutivas antefactuais na elaboração de uma solução informatizada.
\end{abstract}

Palavras-chave: Teoria de Conciliação de Metas. Teoria da Relevância. Modelação Proativa de Metas. Hipóteses Abdutivas Antefactuais. Algoritmos.

\section{OS ALGORITMOS E AS SOLUÇÕES INFORMATIZADAS}

Algoritmos são sequências de etapas que, seguidas na ordem pré-definida, conduzirão à solução de um problema ou atenderão a uma necessidade. Em sentido mais amplo, um algoritmo também pode ser empregado como sinônimo de receita, processo, método, procedimento ou rotina. Tecnicamente, "os algoritmos consistem em uma sequência lógica, ordenada e finita de instruções executáveis que resolvem um determinado problema" (BORATTI, 1990).

O desenvolvimento de um algoritmo fundamenta-se na lógica de programação em um processo que se resume, basicamente, na geração de conclusões a partir de uma lista de premissas ${ }^{2}$. O algoritmo é o ponto de partida para a consecução de um programa e, consequentemente, a base de uma solução informatizada. Em outras palavras, é a partir deles que todas as soluções informatizadas são geradas. 
A qualidade de um algoritmo concorre para a qualidade da solução informatizada. Se o algoritmo é inconsistente, apresentando falhas, então o programa também conterá falhas ${ }^{3}$. Logo, tão importante quanto programar é saber construir um algoritmo de forma adequada. Conforme argumentam Fernandes e Botini (1998, p. 5): "se o algoritmo é de boa qualidade, não importa a linguagem de programação em que ele será desenvolvido, pois o resultado (programa) será consequentemente também de boa qualidade".

O modo de fazer de uma receita de bolo pode ser um exemplo de algoritmo: Misture os ingredientes; Bata por 5 minutos; Unte a forma com manteiga; Leve a forma ao forno; Asse por 30 minutos; Retire do forno; Sirva. Nesse exemplo, é possível perceber que a ordem sintática das ações é importante para a consecução do bolo. Se essa ordenação não é seguida, corre-se o risco de colocar o bolo para assar antes de ter misturado os ingredientes.

Visualizar os algoritmos como uma sequência lógica de etapas ordenadas, finitas e não ambíguas, com um início, um meio e fim, pode levar à suposição de que se trata de um processo totalmente dedutivo, formado a partir de sequências de enunciados (premissas) que levariam a uma determinada conclusão.

A estrutura interna dos algoritmos mostra que realmente é seguido o caminho lógico dedutivo onde: sendo $P$ o antecedente e $Q$ o consequente, dado $P$, teremos Q. Expondo de uma forma mais detalhada teríamos.

$\begin{array}{ll}\mathrm{P} \rightarrow \mathrm{Q} & \mathrm{P} \text { verdadeiro implica } \mathrm{Q} \text { verdadeiro } \\ \mathrm{P} & \text { Dado um } \mathrm{P} \text { verdadeiro } \\ \mathrm{Q} & \text { Por consequência, } \mathrm{Q} \text { será verdadeiro }\end{array}$

Entretanto, ao analisar o processo de elaboração do algoritmo como um todo, percebe-se que parte substantiva da tarefa, quem sabe a parte mais importante, foi omitida, ou seja, a estrutura dos algoritmos pouco ou nada revela como essas premissas foram escolhidas.

Considerado esse contexto, este artigo propõe-se a descrever e explicar, conforme a Teoria da Conciliação de Metas proposta por Rauen (2013), uma situação cotidiana na área da informática: a elaboração de uma solução informatizada baseada em um algoritmo. O estudo parte do pressuposto de que a busca de uma solução informatizada parece ser um processo preliminarmente abdutivo, portanto hipotético, e de que esse processo prévio desencadeia as diversas etapas baseadas no raciocínio dedutivo. 
Antes de tratar o exemplo, apresenta-se a proposta do autor.

\section{MODELAÇÃO PROATIVA DE METAS A PARTIR DE HIPÓTESES ABDUTIVAS}

Rauen (2013) apresenta uma abordagem abdutivo/dedutiva para a modelação proativa de metas que pretende conectar as noções de relevância e de meta. Ele defende a hipótese de que a ampliação do contexto cognitivo para o processamento de metas é abdutiva, de modo que a cognição é movida antes por uma conclusão presumida (meta) do que pela emergência de premissas. Sua proposta não descarta a modelagem dedutiva proposta pela Teoria da Relevância (TR), mas a integra como coadjutora do processo de checagem dessas hipóteses abdutivas.

O modelo de Rauen fundamenta-se especialmente no princípio cognitivo de relevância de que a mente humana tende a maximizar a relevância (SPERBER; WILSON, 1995). O termo relevância é usado para definir uma propriedade dos inputs direcionados à cognição. É através do processamento de um input que podem ocorrer modificações ou reorganizações de suposições cognitivas. Um input é relevante quando vale a pena ser processado, ou seja, quando os efeitos cognitivos superam os esforços de seu processamento. Dessa forma, em contextos iguais, a relevância é maior quando maiores forem os efeitos cognitivos e/ou quanto menores forem os esforços de processamento.

Rauen concorda com Lindsay e Gorayska, quando as autoras afirmam que relevância é um predicado dependente de meta (2004, p. 69). Dessa maneira, os indivíduos atribuem relevância aos inputs baseados nos propósitos ou fins a que se destinam. Isso implica dizer que um evento é relevante quando concorre para algum plano que é suficiente para alcançar uma meta, seja ela cognitiva ou final.

Isso em tudo converge com Silveira e Feltes (1999, p. 37), quando as autoras afirmam que os indivíduos prestam atenção a estímulos que, de alguma forma, vêm ao encontro de seus interesses ou se ajustam às circunstâncias do momento. Assim, os indivíduos tanto podem estar reagindo a estímulos como, de maneira proativa, podem estar agindo por interesses ou propósitos prévios.

A modelação proativa de metas proposta por Rauen (2013) compõe-se de quatro estágios: formulação de meta, formulação de uma hipótese abdutiva antefactual, execução e checagem. 


\subsection{Formulação da meta}

O primeiro estágio do modelo é o da formulação de uma meta. Segundo Rauen, essa etapa pode ser assim formalizada:

[1] O indivíduo $i$ formula uma meta $Q$ no tempo $t_{1}$,

O modelo expõe que o processo se inicia em $t_{1}$, que representa o tempo da emergência da necessidade de atingir a meta $Q$, e a meta $Q$ é uma possibilidade futura ainda não existente no tempo $t_{1}$ (tempo da formulação da meta $Q$ ).

O output desse estágio pode ser representado da seguinte forma.

[1]

$Q$

\subsection{Formulação de uma hipótese abdutiva antefactual.}

O segundo estágio da modelação consiste na proposição de uma hipótese abdutiva antefactual.

Considerando o caso de uma meta $Q$ qualquer e um indivíduo i que se imagina estar nesse estado de meta $Q$ no futuro, $x$ é $Q$ e equivale a um estado $x$ qualquer que satisfará a expectativa de se atingir a meta $Q$. Portanto, o indivíduo $i$ formula uma hipótese abdutiva de que há uma conexão nomológica entre $P$ e $Q$, e considera uma ação antecedente $P$ como pelo menos suficiente para atingir $Q$. Segue-se que $x$ é $P$, e o indivíduo i executa a ação $P$ na expectativa de atingir $Q$.

Nesse estágio, o agente abduz uma hipótese ou inferência à melhor solução, princípio de plausibilidade, que simultaneamente é a solução com menor custo diante do efeito fixo da meta, princípio de relevância. Neste ponto, o modelo converge com o que Harman (1965) chamou de inferência à melhor explicação. A melhor solução estaria vinculada a um princípio de plausibilidade. Desse modo, se uma hipótese abdutiva explicativa $H_{e}$ é aceita quando explica as evidências e nenhuma outra hipótese rival o faz tão bem como $H_{e}$ faz; então, uma hipótese abdutiva antefactual $H_{a}$ é adotada quando sugere atingir uma meta com mais eficiência e nenhuma outra hipótese rival faz isso tão bem como $H_{a}$.

Simultaneamente, Rauen defende que o princípio de relevância assume papel de linha de corte para a emergência de hipóteses rivais tal que a primeira hipótese abdutiva considerada consistente com esse princípio é a hipótese que demanda 
menor custo de processamento diante do efeito fixo de se alcançar a meta. Em outras palavras, no segundo estágio do modelo, o agente formula uma hipótese abdutiva antefactual que melhor concorre para a consecução da meta e que, provavelmente, é a primeira que emergiu diante do contexto. Esse estágio pode ser assim formulado.

[2] $\bigcirc$ indivíduo $i$ abduz uma hipótese antefactual $H_{a}$ para atingir a meta $Q$ em $t_{2}$.

O output desta etapa pode ser assim representado:

$\begin{array}{lll}{[1]} & & Q \\ {[2]} & P & Q\end{array}$

\subsection{Execução}

No terceiro estágio, o agente executa a ação antecedente $P$ que concorre para a consecução da meta $Q$.

Esse estágio pode ser assim formulado.

[3a] O indivíduo $i$ executa $P$ para atingir $Q$ num tempo $t_{3}$, ou

[3b] O indivíduo $i$ não executa $P$ para atingir $Q$ num tempo $t_{3}$.

Essa descrição considera que: há um tempo próprio $t_{3}$ da execução da ação que sucede a formulação da hipótese abdutiva antefactual $H_{a}$; há um modelo positivo no qual a ação $P$ é executada e que, por definição, pode fazer emergir o modelo negativo no qual a ação $P$ não é executada, ou seja, apesar da plausibilidade da hipótese, há contextos onde a ação não é possível ou, mesmo sendo possível, não é executada.

O output do terceiro estágio pode ser formulado da seguinte maneira.

$\begin{array}{lll}{[1]} & & \mathrm{Q} \\ {[2]} & \mathrm{P} & \mathrm{Q} \\ {[3]} & \mathrm{P} & \end{array}$

\subsection{Checagem}

Finalmente, no quarto estágio, ocorre a checagem dedutiva da formulação hipotética.

[4a] Considerando-se [2] "Se $P$, então $Q$ " e [3a] $P$, o indivíduo $i$ atinge $Q^{A}$ num tempo $t_{4}$, ou 
[4b] Considerando-se [2] "Se $P$, então $Q$ " e [3b] $\neg P$, o indivíduo $i$ atinge $\neg Q$ ' num tempo $t_{4}$, tal que:

O quarto estágio consiste na avaliação da (in)ação antecedente $P$ no escopo dedutivo da formulação hipotética "Se $P$, então $Q$ ", o que conflui com o módulo dedutivo de Sperber e Wilson $(1995)^{5}$. Desse modo, quer o indivíduo opte ou não pela consecução da ação antecedente $P$, as consequências serão avaliadas dedutivamente dentro do escopo da hipótese abdutiva.

O output do quarto estágio em [4] pode ser visto a seguir:

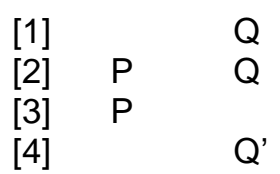

Considerando o estágio de checagem, o autor propõe quatro tipos de consecução conforme as noções de conciliação dos resultados com a meta e de confirmação da hipótese: conciliação ativa, inconciliação ativa, conciliação passiva e inconciliação passiva.

Além disso, esses tipos de consecução levaram o autor a propor uma modelação de enunciados hipotéticos "Se $\mathrm{P}$, então Q". Rauen propõe a hipótese de que, por padrão, hipóteses abdutivas emergem como categóricas $(P \Leftrightarrow Q)$ e modelam conciliações ativas. Diante de problemas ou dilemas, essa formulação torna-se bicondicional $(P \leftrightarrow Q)$, admitindo inconciliações passivas. Quando $P$ é apenas suficiente, a formulação torna-se condicional $(P \rightarrow Q)$, admitindo conciliações passivas. Quando $P$ é necessário, mas não garante $Q$, a formulação torna-se habilitadora $(P \leftarrow Q)$, admitindo inconciliações ativas. Por fim, quando todas as possibilidades são plausíveis a formulação torna-se tautológica $(P-Q)$.

Conhecida a modelação de Rauen em linhas gerais, segue a análise de uma situação rotineira envolvendo solução informatizada em que um usuário solicita ao profissional de informática o desenvolvimento de um programa.

\section{MODELAÇÃO PROATIVA DE UM PROGRAMA}

Considere o caso de um usuário que solicita a um profissional da área de informática um programa que gere um relatório impresso, em ordem alfabética, com nome e telefone de todos os seus fornecedores. 


\subsection{Formulação da meta}

Conforme a proposta de Rauen, o primeiro estágio consiste na formulação de uma meta. No caso em destaque, a meta poderia ser assim formulada:

[1] O programador $i$ formula a meta $Q$ de gerar um relatório em $t_{1}$.

No caso, o profissional responsável para elaboração da solução informatizada $i$ formula/identifica o problema/a necessidade $Q$ do usuário em um determinado tempo $t_{1}$, tal que em $t_{1}$ o relatório não existe concretamente.

O output desse estágio é o que segue.

Q gerar relatório, programador

\subsection{Formulação de uma hipótese abdutiva antefactual}

Num segundo estágio, após definida a meta $Q$, parte-se para a escolha do melhor caminho para se desenvolver a solução. Conforme Rauen (2013), estamos diante de um processo onde é elencada a hipótese abdutiva antefactual, ou seja, o caminho mais plausível para se chegar à meta $Q$ em $t_{1}{ }^{6}$.

No exemplo, o profissional escolhe a sequência algorítmica (antecedente $P$ ) que surge como a inferência à melhor solução, ou seja, que emerge como proposta minimamente suficiente para atingir o consequente $Q$ que é gerar relatório ${ }^{7}$.

[2a] O programador $i$, abduz a melhor hipótese antefactual $H_{a}$ para atingir a meta $Q$ de gerar o relatório em $t_{2}$.

Para que o output desta etapa esteja completo considera-se aqui que o programador $i$ tenha inferido como melhor hipótese abdutiva antefactual $\left(H_{a}\right)$ uma sequência algorítmica do tipo: leitura dos dados, ordenação dos dados, visualização do relatório e impressão do relatório (doravante sequência ${ }_{1}$ ). Ao escolher esta hipótese 0 indivíduo considera que a hipótese $H_{a}$ pode ser mapeada por uma formulação hipotética do tipo "Se $P$, então $Q$ ", segundo a qual se uma ação antecedente $P$ for executada, então um estado consequente $Q$ pode ser atingido. Portanto, se todos os passos do algoritmo forem executados, então será gerado o relatório. Além disso, a abdução desta hipótese faz supor que ela foi considerada 
pelo individuo i como a mais plausível e suficiente para gerar o relatório e a de mais baixo custo de processamento diante do efeito fixo de gerar o relatório.

O resultado desse estágio pode ser visto a seguir.

[2b] Se o programador $i$ executar a sequência ${ }_{1} P$, então será gerado o relatório $Q$ em $t_{2}$.

O resultado do processamento de [2b] pode ser representado desta forma.

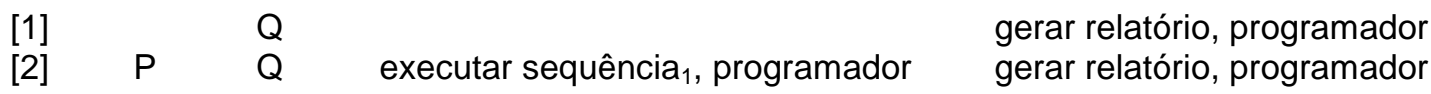

\subsection{Execução}

Abduzida a melhor hipótese, desencadeia-se o terceiro estágio que consiste na provável execução da ação antecedente $P$, ou seja, formula-se um algoritmo composto por: leitura dos dados, ordenação dos dados, visualização do relatório e impressão do relatório. Veja-se a versão positiva dessa formulação.

[3] O indivíduo $i$ executa a sequência ${ }_{1} P$ para atingir a meta de geração de um relatório $Q$ num tempo $t_{3}$.

O output desta terceira etapa é o que segue:

$\begin{array}{llll}{[1]} & & \mathrm{Q} & \\ {[2]} & \mathrm{P} & \mathrm{Q} & \begin{array}{l}\text { executar sequência } \\ \text {, }\end{array} \text {, programador } \\ {[3]} & \mathrm{P} & & \text { executar sequência }, \text { programador }\end{array}$

gerar relatório, programador gerar relatório, programador

\subsection{Checagem}

Uma vez executada a ação do terceiro estágio, versão positiva do modelo, inicia-se o quarto estágio que consiste na checagem dedutiva da formulação hipotética:

[4] Considerando-se [2] "Se $P$, então $Q$ " e [3] $P$, o programador $i$ atinge $Q$ ' num tempo $t_{4}$, onde:

Após a execução do processo, o programador obtém um resultado concreto da ação, ou seja, o que antes era apenas uma meta, agora é um resultado identificado como $Q$ '. Sendo um resultado alavancado por uma meta $Q$, partindo da perspectiva de que "Se $P$, então $Q$ ", porque ele é identificado como $Q$ ' e não como $Q$ ? A 
apresentação do resultado como Q' explica-se pelo fato de que o resultado efetivo de um processo sempre será de alguma forma diferente do que foi imaginado mentalmente, por maior que tenha sido o rigor da execução das etapas. Além disso, Q e Q' pertencem a tempos distintos de processamento, $t_{1}$ e $t_{4}$ respectivamente.

Neste estágio, ocorre uma avaliação da ação antecedente $P$ no escopo dedutivo da formulação hipotética "Se $P$, então $Q$ ". Assim, no cenário positivo, avalia-se a geração concreta do relatório com base na formulação algorítmica dasequencia ${ }_{1}$. Este estágio tem como saída os seguintes possíveis resultados:

$\begin{array}{llll}{[1]} & & Q & \\ {[2]} & P & Q & \begin{array}{l}\text { executar sequência } \\ \text { executar sequência } \text {, programador }\end{array} \\ {[3]} & P & & \text { exector } \\ {[4]} & & Q^{\prime} & \end{array}$ gerar relatório, programador gerar relatório, programador geração do relatório, programador

\section{CONSIDERAÇÕES FINAIS}

A formulação de algoritmos é a base para o desenvolvimento de soluções informatizadas. Trata-se de uma "sequência de raciocínios ou operações que oferecem a solução de certos problemas" (MICHAELIS, 2013). Posto isso, conforme Fernandes e Botini (1998, p. 39), o problema/a necessidade é o ponto de partida na busca de soluções informatizadas: "construir algoritmos consiste em estruturar um processo de forma lógica, precisamente definido e optando sempre pelo melhor caminho".

Considerando hipóteses abdutivas antefactuais e a modelação proativa de metas, conforme modela Rauen (2013), somos incitados a visualizar os algoritmos sob a perspectiva de um ponto de partida que ocorre antes mesmo de sua formulação concreta. Isto concorre para questionar se, de fato, as premissas guiam a formulação de um algoritmo. Argumenta-se que há um processo prévio onde o foco está na meta a ser alcançada. Desta forma, e antes mesmo de se dispor a elaborar um algoritmo, o programador considera a meta $Q$ como guia para a escolha do melhor caminho para alcançá-la. A escolha das melhores hipóteses abdutivas antefactuais, o que Rauen (2013) chamou de inferência à melhor solução, é justamente o processo fundamental que antecede mesmo a sequência dedutiva de etapas. Voltando ao exemplo do algoritmo do bolo, as etapas envolvidas como misturar os ingredientes e levar ao forno, parecem não ser o que alavanca todo o 
processo e sim o objetivo, a meta, que é fazer um bolo. Ou seja, após a visualização da meta "o bolo" é que se desencadeia o algoritmo. Analogicamente, a modelação proativa de metas antecede a elaboração de qualquer solução informatizada.

O exemplo da solução para gerar um relatório, retrata uma situação que é comum quando estamos tratando da elaboração de soluções informatizadas. A busca pela solução não é do profissional da informática (programador, desenvolvedor, analista,...), mas de um terceiro, aqui identificado como usuário. Ao perceber uma necessidade/um problema, o usuário solicita ao profissional que desenvolva uma solução. Portanto, estamos diante de um processo que tem origem na comunicação entre quem está buscando uma solução e quem irá desenvolvê-la. O sucesso desta solução está relacionado ao desenvolvimento deste processo comunicativo, que é preliminar a todas as outras etapas.

Ao enunciar o problema, além de já tê-lo identificado, o usuário precisa verbalizar o que ele visualiza mentalmente como possível solução, ou seja, ele precisa expor esta "imagem mental da situação", de forma que seu ouvinte entenda o que ele deseja, a sua meta $Q$. À pessoa responsável pela busca da solução compete interpretar qual a necessidade do momento para elaborar uma possível solução e isto é feito a partir da identificação da meta $Q$ '.

Isso só é possível porque o cérebro humano é capaz de imaginar soluções, mesmo antes de elas existirem concretamente. Desta forma a busca de soluções é desencadeada pela imaginação de um resultado, e esse processo criativo é mais bem formalizado de modo abdutivo. Veja-se:

$\begin{array}{ll}Q & \text { Q é a meta ou a conclusão que se busca } \\ P \rightarrow Q & \text { P verdadeiro implica } Q \text { verdadeiro } \\ P & \text { P é verdadeiro }\end{array}$

Conforme essa formulação, o processo se inicia do consequente $Q$, ou seja, da meta a ser atingida. No caso, trata-se de algo que de fato ainda não existe, mas é imaginado no futuro. Uma vez que as pessoas que imaginam a solução e as que desenvolvem são diferentes, isso implica sérios obstáculos comunicacionais. Certamente pode haver situações em que a solução imaginada $Q$ e a solução desenvolvida $Q$ ' sejam muito próximas e quanto mais próximas eles forem, melhor terá sido o resultado obtido. Esse tipo de resultado tanto será melhor quanto melhor forem as capacidades dos profissionais de abduzirem melhores soluções. 
Essa constatação ressalta que se as máquinas operam exclusivamente por dedução (daí a necessidade de os algoritmos serem dedutivos), o cérebro humano funciona abdutivo/dedutivamente. Talvez este seja um dos motivos que faz com que máquinas e seres humanos se diferenciem. Nas palavras de John R. Lucas (apud SEARLE, 1998, p. 87) é impossível negar a diferença entre máquinas e homens:

[...] nossa compreensão supera a de qualquer computador. Um computador utiliza apenas algoritmos. Logo, quando um computador corrobora um teorema ele tem de usar um algoritmo que comprove teoremas. No entanto, há algumas proposições que percebemos que são verdadeiras, mas não são comprováveis no sistema. Elas, consequentemente, não são teoremas do sistema e, por conseguinte, não podem ser corroboradas por um algoritmo que comprove teorias. [...] deduz-se que nosso conhecimento dessas verdades não pode ser algorítmico. Mas, como os computadores só utilizam algoritmos, conclui-se que não somos computadores.

\section{NOTAS}

${ }^{1}$ Mestre em Extensão Rural pela Universidade Federal de Santa Maria (UFSM). Doutoranda em Ciências da Linguagem pela Universidade do Sul de Santa Catarina (UNISUL). Docente do Ensino Básico, Técnico e Tecnológico do Instituto Federal Catarinense (IFC/Campus Sombrio). E-mail: sandravieiraifc@hotmail.com.

2 John McCarthy (1958) foi quem primeiro propôs o uso da lógica matemática para a programação de computadores. Muitas linguagens artificiais foram elaboradas a partir deste conceito na programação.

${ }^{3}$ Segundo Teixeira (2004), uma pesquisa feita pela empresa de consultoria americana Cutter, com 150 produtoras de software, apontou que um terço delas admitiu lançar no mercado softwares com defeitos. Além disso, a economia americana gasta aproximadamente 60 bilhões de dólares por ano com softwares defeituosos. No Brasil, especialistas acreditam que este valor pode chegar a 8 bilhões de reais, ou seja, $0,6 \%$ do PIB.

${ }^{4}$ Conforme Rauen (2013, p. 9), a expressão Q' representa o fato de que a consecução da meta é sempre em alguma medida diferente de sua projeção.

${ }^{5}$ No mecanismo de interpretação guiado pelo conceito de relevância, destaca-se o módulo dedutivo proposto por Sperber e Wilson (1995). Trata-se de um módulo que captura suposições obtidas da percepção ou da memória e deduz conclusões de modo não trivial e não demonstrativo por regras de eliminação do tipo eliminação-e, eliminação-ou e modus ponens. Segundo Rauen (2013), este mecanismo tem se mostrado eficiente na modelação dos processos cognitivos envolvidos na interpretação de inputs verbais. Para o autor, isto se deve ao fato de que o indivíduo busca uma interpretação que satisfaça sua expectativa de relevância ótima, seguindo uma rota de esforço mínimo e baseando-se na codificação linguística. Na tentativa de obter um significado explícito (explicatura), ele enriquece esses inputs e, eventualmente, consegue obter um significado implícito a partir deste significado explícito (implicatura). $O$ processo se encerra quando a interpretação se conforma com sua expectativa de relevância.

${ }^{6}$ É fato que existem muitas formas de se desenvolver a solução. Segundo Psillos (2002, p. 7)se muitas hipóteses rivais podem explicar um mesmo evento, uma meta também pode ser atingida por muitas soluções, e a abdução não possuiria ferramentas para restringi-las. Contudo, o sucesso como os indivíduos abduzem explicações sugere haver mecanismos para classificar hipóteses por suas virtudes explicativas: o conhecimento enciclopédico, as experiências anteriores, bom senso, contribuem para a classificação de hipóteses ou a emergência de uma única hipótese tomada como a mais plausível, já ela emerge como a mais forte. 
7 Segundo Harman (1969) estaria em funcionamento o principio da plausibilidade, baseado nas virtudes explicativas, fazendo emergir a alternativa algorítmica minimamente suficiente para atingir a meta. Observe-se que a escolha de um caminho que seja suficiente para a consecução de uma meta, não garante que a conclusão seja verdadeira mesmo com premissas verdadeiras. Portanto, elencar uma hipótese abdutiva antefactual pode redundar em fracasso.

\title{
PROACTIVE MODELING OF GOALS AND ELABORATION OF COMPUTERIZED SOLUTIONS
}

\begin{abstract}
Computerized solutions for solving problems that emerge from concrete everyday needs are structured through deductive algorithms. However, even before designing algorithms, there is an abductive step guided by the goal to be reached which is usually neglected. Following Rauen's (2013) Goal Conciliation Theory, I intend in this article to describe and explain how the process of formulation, execution and checking of ante-factual abductive hypotheses occurs in developing a computerized solution.
\end{abstract}

Keywords: Goal Conciliation Theory. Relevance Theory. Proactive Modeling of Goals. Ante-factual Abductive Hypotheses. Algorithms.

\section{REFERÊNCIAS}

BORATTI, I. C. Conceitos básicos de lógica de programação. Florianópolis, UFSC, 02 ago. 1990. Aula ministrada aos alunos do curso de ciências da computação.

CHANDOR, A.; JOHN, G.; WILLIAMSON, R. Análise de sistemas: teoria eprática. Rio de Janeiro: Livros técnicos e científicos, 1977.

FERNANDES, A. L. B.; BOTINI, J. Construção de algoritmos. Rio de Janeiro: SENAC, 1998.

HARMAN, G. The inference to the best explanation. Philosophical Review, v. 74, p. 88-95, 1965.

JOHNSON-LAIRD, P. N.; BYRNE, R. M. J.Conditionals: a theory of meaning, pragmatics, and inference. Psychological Review, v. 109, n. 4, p. 646-678, 2002. 
LINDSAY, R.; GORAYSKA, B. Relevance, goal management and cognitive technology. In: GORAYSKA, Barbara; MEY, Jacob. Cognition and technology: coexistence, convergence, and co-evolution. Amsterdam: J. Benjamins, 2004.

MICHAELIS. Dicionário de português on-line. Disponível em:

<http://michaelis.uol.com.br/moderno/portugues/>. Acesso em: 15 maio 2013.

PSILLOS, S. Simple the best: A Case for abduction. In: KAKAS, A.C.; SADRI, F. (Eds.). Computational logic: logic programming and beyond. Berlin: Springer-Verlag, 2002. p. 605-626. Disponível em: <http://www.phs.uoa.gr/ psillos/>. Acesso em: set. 2013.

RAUEN, F. J. Hipóteses abdutivas antefactuais e a modelação proativa de metas. Signo, Santa Cruz do Sul, v. 38, n. 65, p. 188-204, jul./dez. 2013. Disponível em: <http://online.unisc.br/seer/index.php/signo>. Acesso em: 18 mar. 2014.

SEARLE, J. R. O mistério da consciência. São Paulo: Paz e Terra, 1998.

SILVA, C. R. da. Conversão de registros de representação: desenvolvimento de aplicativos para o ensino-aprendizagem de funções. 2009. 156 f. Dissertação (Mestrado)-Universidade do Sul de Santa Catarina, Tubarão, 2009. Disponível em: <http://aplicacoes.unisul.br/pergamum/pdf/98800_Cintia.pdf>.

SILVEIRA, J. R. C.; FELTES, H. P. M. Pragmática e cognição: a textualidade pela relevância e outros ensaios. 3. ed. Porto Alegre: Edipucrs, 1999.

SPERBER, D; WILSON, D. Relevance: communication \& cognition. 2. ed. Oxford: Blackwell, 1995.

TEIXEIRA JR, Sérgio. O caçador de defeitos. 25 de março de 2004. Disponível em: $<$ http://exame.abril.com.br/revista-exame/edicoes/0814/noticias/o-cacador-dedefeitos-m0054924>. 